\title{
Article
}

\section{Risques sanitaires liés aux modes d'assainissement des excreta à Yaoundé, Cameroun}

\author{
Yongsi H.B. Nguendo ${ }^{1}$, Gérard Salem ${ }^{2}$, Jean-Pierre Thouez ${ }^{3}$ \\ 1 Géographie/Épidémiologie sociale, Laboratoire Développement durable et dynamique territoriale, Département de géographie, \\ Université de Montréal, CP 6128, succursale Centre-ville, Montréal (Québec) H3C 3J7, Canada \\ 2 Géographie de la santé, Directeur du département Observatoire des cancers, Institut national du cancer, 52 avenue André Morizet, \\ 92100 Boulogne Billancourt, France \\ 3 Géographie de la santé, Département de géographie, Université de Montréal, Pavillon Strathcona, 520 Côte Sainte-Catherine, \\ local 305, Montréal (Québec) H2V 2B8, Canada
}

\begin{abstract}
En examinant le lien qui existe entre les conditions d'assainissement du milieu et la prévalence des diarrhées infantiles, cet article montre en quoi les problématiques sur le risque nécessitent une démarche interdisciplinaire. L'épidémiologie et la géographie croisent ainsi leurs données et s'enrichissent mutuellement en montrant en quoi aux données épidémiologiques répondent des variabilités intra-urbaines au sein même des quartiers. La dimension spatiale du risque diarrhéique permet ainsi d'entrevoir les inégalités propres à la distribution des risques, qui est aussi une distribution des facteurs de la pauvreté urbaine.
\end{abstract}

La Rédaction

\begin{abstract}
Mots-clés :
assainissement; morbidité

diarrhéique réelle ; milieu urbain tropical; de mesurer leur prévalence et d'appréhender leur distribution spatiale. Les analyses effectuées sur les géographie de la santé; selles des 3034 enfants examinés ont révélé 437 cas de selles diarrhéiques (soit une prévalence de 14,4 \%). épidémiologie analytique Parmi les nombreux facteurs de risques étudiés, les pratiques d'assainissement des populations concernant les matières fécales se sont révélées être associées à ces diarrhées. De plus, il s'est avéré que les niveaux d'atteinte diarrhéique variaient considérablement d'un quartier à un autre du fait du caractère différencié de l'urbanisation de la ville.
\end{abstract}

\begin{tabular}{|c|c|}
\hline $\begin{array}{l}\text { Keywords: } \\
\text { Cleansing; } \\
\text { diarrhoeas; } \\
\text { tropical urban } \\
\text { environment; } \\
\text { health geography; } \\
\text { analytic epidemiology; } \\
\text { Cameroon }\end{array}$ & $\begin{array}{l}\text { Abstract - Health risks associated with faeces cleansing methods in Yaound } \\
\text { Cameroon. Diarrhoeal diseases are a major public health problem in developing countries. They hav } \\
\text { assumed an endemic and epidemic profile in the developing world as attested by the recent upsurge i } \\
\text { epidemics of (a) cholera in Angola and Cameroon, (b) dysentery in Sudan, Central African Republi } \\
\text { Bolivia, Paraguay, and (c) typhoid fever in Democratic Republic of Congo and Haiti. Striking recen } \\
\text { evaluations show that they are a major cause of infant mortality between the weaning age and fiv } \\
\text { years: } 1.8 \text { million infants have died from diarrhoeas in 2005. From an aetiological and epidemiologica } \\
\text { perspective, they are associated with poor and unhealthy living conditions. This situation motivated } \\
\text { cross-sectional study relating to children aged } 6 \text { to } 59 \text { months, which was carried out in May } 2002 \text { i } \\
\text { Yaoundé (Cameroon). The objectives of the study were to identify risks factors of diarrhoeal diseases in th } \\
\text { city, to measure their prevalence and to grasp their spatial distribution. Field investigation comprised tw }\end{array}$ \\
\hline
\end{tabular}

Auteur correspondant : Y.H.B. Nguendo, nguendoyongsi_cm@yahoo.fr 
a prevalence of $14,4 \%$ (437 diarrhoea cases among the 3034 examined children). Among the risk factors investigated, cleansing practices of faeces proved to be strongly associated with these diarrhoeas. Moreover, it has appeared that levels of diarrhoeal attack varied considerably from one district to another because of disparities in the urbanization process of Yaoundé metropolis.

\section{Introduction}

L'assainissement est l'action ou la somme des actions visant à améliorer toutes les conditions qui, dans le milieu physique de la vie humaine, influent ou sont susceptibles d'influer défavorablement sur le bien-être physique et social des populations. Ces actions ont pris de l'ampleur avec la transformation des sociétés occidentales. De fait, la croissance continue de la population et l'industrialisation effrénée ont entraîné l'abandon dans la nature de rejets constitués de matières solides et d'eaux résiduaires, à l'impact négatif sur la santé ${ }^{1}$. Longtemps, l'ampleur de cet impact fut négligeable, puisque les populations vivaient très dispersées et que les rejets s'intégraient dans la nature et étaient éliminés au cours du cycle biologique. Mais, avec l'urbanisation rapide des $\mathrm{XIX}^{\mathrm{e}}$ et $\mathrm{XX}^{\mathrm{e}}$ siècles, les nouvelles conditions de vie ont généré pollutions et nuisances de tous ordres (Giacottino, 1979). Dans les villes en développement, où l'urbanisation a été spectaculaire et non maîtrisée par les pouvoirs publics ${ }^{2}$ (Amat-Roze, 1983), les problèmes posés par les rejets solides et liquides en milieu urbain sont nombreux (Bley et al., 1997). Ils procèdent de la pollution du sol et des nappes souterraines comme de la collecte et de l'évacuation des eaux usées. $C^{\prime}$ est pourquoi la maîtrise de l'assainissement au sens large est de nos jours l'un des défis majeurs des villes en développement.

À Yaoundé, ce défi est loin d'être satisfait, car la situation au niveau de l'évacuation des urines et des matières fécales y est décevante. De fait, le développement accéléré et incontrôlé de la ville a entraîné une prolifération des systèmes autonomes de collecte et d'évacuation des excreta dans les différents quartiers de la capitale, où ces systèmes ont un impact considérable sur la santé des populations par le biais des ressources en eau qu'elles utilisent (Tanawa et al., 2002). À cet égard, les modes d'assainissement mis en place ne peuvent plus être considérés seulement comme des commodités, mais comme des nuisances, car les risques sanitaires qu'ils font courir aux citadins sont assez élevés, ainsi que l'attestent les maladies diarrhéiques qui y sont associées.

\footnotetext{
${ }^{1}$ Cette étude procède d'un travail interdisciplinaire et interuniversitaire initié par l'Institut de recherche pour le développement (IRD, Paris) et mené sur le terrain par 1'UR093, Populations et espaces à risques sanitaires (PERSAN).

2 Pour autant qu'on admette qu'ils sont les principaux canalisateurs des actions des populations, à travers la définition d'une politique d'habitat, de gestion des villes ou de création de normes en matière d'assainissement, par exemple.
}

\section{Mise en contexte et objectif de l'étude}

Les maladies diarrhéiques sont depuis très longtemps connues des civilisations. Elles ont été décrites dès la haute Antiquité grecque comme étant une "maladie dans laquelle les intestins ne retiennent rien, et où l'on rend presque tout sur le champ les aliments tels qu'on les a pris et sans être digérés » (Gallien [129-201], traduit par Daremberg, 1854). Les fondements d'une hygiène défectueuse comme origine des diarrhées étaient ainsi posés. Ils vont rester sous-jacents à toutes les autres causes possibles pendant des siècles. Vers le milieu du XVII ${ }^{\mathrm{e}}$ siècle, les diarrhées sont nommées maladies «humoristes » et sont classées dans la catégorie des maladies des intestins au même titre que les ulcères et la dysenterie. Les décès attribuables à la maladie allant s'amplifiant, les diarrhées sont appréhendées comme un véritable fléau au même rang que la peste et la maladie du charbon (Colson, 1808). Elles vont durablement marquer tout le XVIII ${ }^{\mathrm{e}}$ siècle, tant par les souffrances engendrées que par les dramatiques épidémies occasionnées, notamment au sein des armées où les conditions hygiéno-diététiques étaient généralement précaires (Dufour, 1814). C'est à partir de cette époque que les maladies diarrhéiques seront véritablement considérées comme le reflet de la médiocrité des conditions matérielles d'existence. Cette compréhension somme toute judicieuse des faits oriente, bien sûr, les médecins vers la prescription de thérapies curatives (Dupont, 1952), mais surtout vers une stricte observance de règles d'hygiène de vie individuelle et collective (Ferrière, 1992). Ceci va contribuer au recul de la pathologie, du moins dans le monde occidental où elle a été décrite en premier lieu, car, de nos jours, les maladies diarrhéiques demeurent un problème majeur de santé publique dans les pays en développement. Certes, on sait qu'elles y sévissaient déjà à l'état endémique et que, périodiquement, elles donnaient lieu à de vastes flambées épidémiques (Nielly, 1881), mais l'absence d'informations ne permet malheureusement pas d'apprécier leur évolution depuis la découverte du premier vibrion cholérique, en Égypte, en 1883 jusque vers le milieu du $X^{\mathrm{e}}$ siècle. Bien que les informations concernant leur incidence et leur prévalence soient parfois imprécises, les diarrhées ont toujours été décrites comme un des plus grands fléaux qui touchent en permanence les jeunes enfants sous les Tropiques (Gentilini et Duflo, 1986). Malgré leur imprécision, les statistiques en font une cause importante de mortalité chez l'enfant entre le moment du sevrage et l'âge de 5 ans (Snyder et Merson, 1982 ; OMS, 2001). Les évaluations récentes sont 
saisissantes : 1,5 million d'enfants en seraient morts en 1999 sur une proportion d'exposés de l'ordre de 338 millions, dont 209 millions en Asie (Chine exclue), 75 millions en Afrique et 54 millions en Amérique latine (Imbert, 2001; OMS, 2006). Toutefois, si la morbidité est réelle sous toutes les latitudes, la létalité concerne presque exclusivement les pays en développement, où un enfant présente en moyenne 3 à 9 épisodes diarrhéiques par an et où cette pathologie constitue le premier motif d'hospitalisation en milieu pédiatrique (Dennehy, 2005 ; Addy et al., 2004). Il en est ainsi du Cameroun, où la littérature médicale rapporte que les maladies diarrhéiques sont la première cause de morbidité et de mortalité infantiles. Plus encore que ce classement général, c'est le pourcentage de 13,9\% pour des zones urbaines comme Douala et Yaoundé qui attire l'attention (MINPAT, 1998). C'est forts de ce constat que nous avons entrepris ce travail dont l'objectif est double : objectiver les modes d'assainissement en urines et matières fécales comme facteurs de risques associés à la pathologie dans la ville; relever les variations spatiales des taux de prévalence.

\section{Matériel et méthodes}

\section{Cadre de l'étude}

Notre étude porte sur la ville de Yaoundé, qui se trouve en Afrique centrale, sous des latitudes tropicales légèrement au-dessus de l'équateur. Plus précisément, la ville est localisée entre $3^{\circ} 47^{\prime}$ et $3^{\circ} 56^{\prime} \mathrm{N}$ et entre $11^{\circ} 10^{\prime}$ et $11^{\circ} 45^{\prime} \mathrm{E}$. Située à environ $250 \mathrm{~km}$ de la côte de l'océan Atlantique et à la lisière de la grande forêt du Sud, elle s'inscrit dans le bassin du Mfoundi sur une étendue d'environ $256 \mathrm{~km}^{2}$ (Fig. 1). Les données météorologiques montrent que Yaoundé est soumis à un climat de type équatorial guinéen classique : précipitations régulières et abondantes (1 $600 \mathrm{~mm} / \mathrm{an})$; température moyenne annuelle de $23^{\circ} \mathrm{C}$; amplitude thermique annuelle faible de $2^{\circ} 4$; existence de quatre saisons - deux saisons sèches (de novembre à mars et de juillet à août) et deux saisons de pluies (de septembre à octobre et d'avril à juin). Le sous-bassement de Yaoundé est un socle ancien principalement gneissique qui a été affecté par une tectonique de grande envergure (Kuété, 1977). De ce fait, les formations superficielles de l'agglomération sont constituées de sols ferrallitiques rouges généralement désaturés. Dans le périmètre urbain actuel de Yaoundé, on trouve une végétation mixte faite de reliques de forêt sur les sommets et les versants des montagnes, de plantations de bananiers et d'arbres fruitiers aux abords des voies publiques et des habitations, de forêts-galeries, de graminées et de plants maraîchers qui tapissent le fonds des vallées. Avec une superficie estimée à 18000 ha, la ville abrite une population évaluée à 1500000 habitants (Ondoua, 1992).

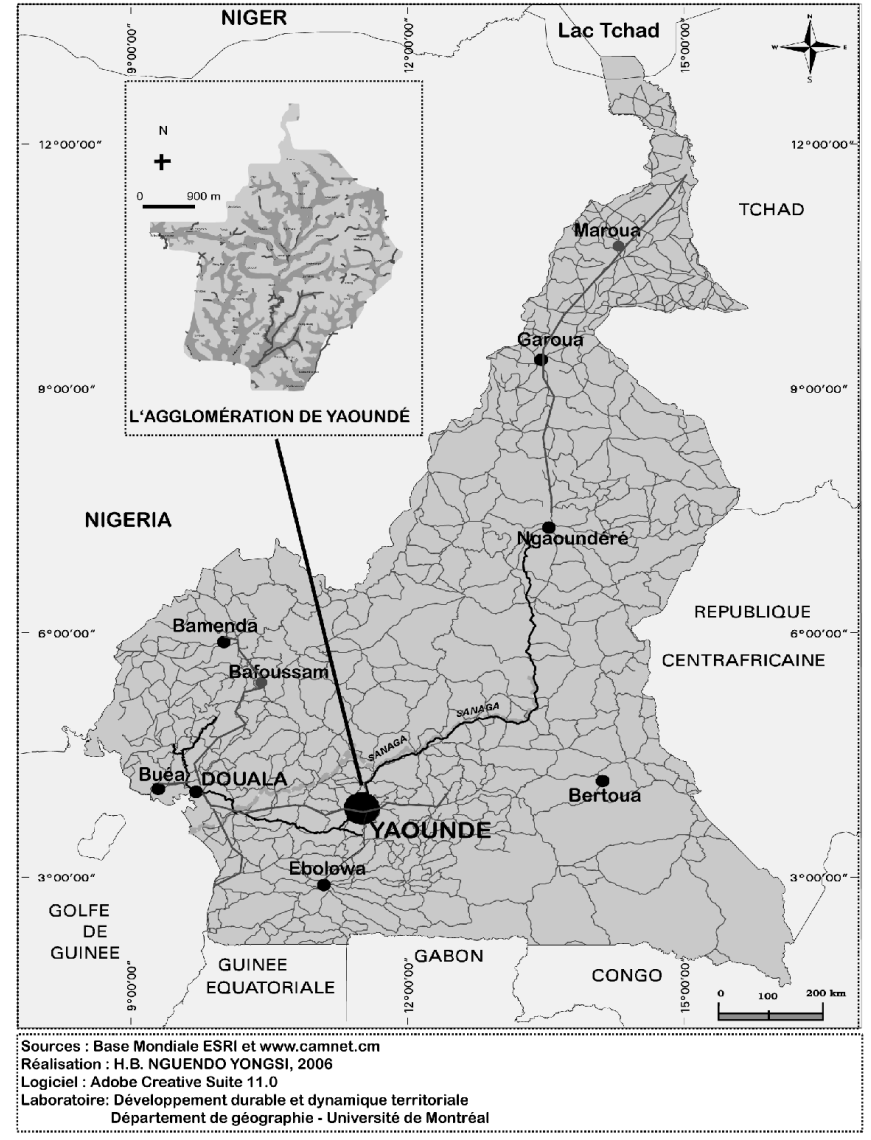

Fig. 1. Localisation de la ville de Yaoundé au Cameroun.

\section{Données et méthodes}

Les données qui sont présentées ici proviennent d'une étude interdisciplinaire menée sur la ville en 2002 par l'Institut de recherches pour le développement (IRD) en collaboration avec le Centre Pasteur du Cameroun (CPC). Centrée sur la santé en milieu urbain, cette étude a rassemblé des géographes, des médecins, des épidémiologistes et un démo-statisticien. L'étude s'est déroulée durant les mois de mai et juin, qui correspondent à la grande saison des pluies.

Bien que notre base de sondage ait été composée de tous les quartiers et ménages de la ville, nous avons procédé à un sondage stratifié à deux degrés. Dans un premier temps, nous avons choisi 20 quartiers représentatifs des 105 que compte la ville. Afin d'avoir une taille d'échantillon suffisante pour la validation scientifique de nos résultats, nous avons ensuite tiré au sort 3 034 ménages. Sélectionné parmi les 20 quartiers préalablement déterminés, chaque ménage devait comporter en son sein un enfant âgé de moins de cinq ans, notre population-cible étant les enfants âgés de 6 à 59 mois. Coordonnée par nos soins, cette étude a été exécutée par des équipes de médecins en fin de formation à la faculté de médecine et des sciences biomédicales de l'Université 
de Yaoundé I et par des enquêteurs attitrés de l'Institut de formation et de recherche démographiques (IFORD) de Yaoundé. Après plusieurs jours de stages théoriques et pratiques, ces équipes ont visité les ménages sélectionnés pour recueillir, à l'aide de questionnaires standardisés, les données relatives aux deux volets de cette étude, à savoir :

- le volet socio-environnemental : identification et caractérisation des conditions de vie domestique et péridomestique des ménages;

- le volet médical : approuvé par le Comité national d'éthique du Cameroun, il s'adressait uniquement à l'enfant de moins de 5 ans du ménage. Hormis l'appréciation des antécédents médicaux, ce volet consistait principalement à prélever les selles des enfants dans le but de détecter les cas de diarrhées. Ainsi, lorsqu'un cas de diarrhée était déclaré lors du passage des médecins de l'équipe ou durant les 15 jours précédents, un échantillon de selles était prélevé et acheminé, selon les exigences en la matière, dans les différents laboratoires (de bactériologie, virologie et parasitologie) du Centre Pasteur pour confirmation et identification des germes causaux ${ }^{3}$. Une fois les résultats des différentes analyses médicales obtenus, chaque échantillon de selles confirmé positif était relié aux données sociodémographiques du ménage auquel l'enfant appartenait et aux données environnementales de son espace de vie. Les fichiers de données ainsi constitués ont été ensuite intégrés dans un système d'information géographique (SIG) à des fins d'analyse spatiale. Nous avons réalisé une analyse statistique bivariée accompagnée de tests de significativité pour vérifier le niveau $\mathrm{d}^{\prime}$ association entre nos deux principales variables (dispositif d'assainissement et diarrhée). Les logiciels qui ont été utilisés au cours de ces différents processus sont Epi Info 3 (enregistrement des données brutes, vérification et validation des informations collectées), SPSS 10.0 (analyse statistique et tabulation) et ArcInfo 8.2 (modélisation spatiale).

\section{Résultats et commentaires}

Par urines et matières fécales, nous entendons les déjections humaines (excreta) qui rentrent dans la terminologie générale des eaux usées. En raison de leur nature et de leur origine, toutes ces eaux sont souillées. Aussi doivent-elles

\footnotetext{
${ }^{3}$ Afin d'éviter la confusion entre des selles liquides qui seraient la manifestation d'une allergie à un aliment ou à un médicament quelconque et des selles proprement diarrhéiques, des examens macroscopique et microscopique étaient faits sur chaque échantillon de selle prélevée pour s'assurer que le diagnostic établi correspondait bien à notre définition opérationnelle de la diarrhée, à savoir : émission en trop grande quantité de selles liquides, molles ou pâteuses depuis 3 à 5 jours consécutifs, accompagnée de colique, ténesme et fièvre.
}

être collectées, traitées et rejetées, une fois assainies, dans le milieu récepteur. À cet égard, il importe de signaler que Yaoundé ne dispose pas d'un réseau général d'assainissement. La population urbaine, dans sa grande majorité ${ }^{4}$ utilise alors des systèmes individuels d'assainissement qui, à cause de l'inexistence ou des dysfonctionnements des stations d'épuration, rejettent les eaux usées directement dans la nature, comme d'ailleurs tous leurs déchets (Ngwe, 1999).

\section{Des modes d'assainissement variés}

Notre enquête montre qu'en dehors du système d'évacuation collectif des cités populaires planifiées, l'autoassainissement reste le principal mode d'évacuation des matières fécales dans la ville. Les techniques ou dispositifs utilisés par les populations sont divers. Ils vont des simples trous creusés dans le sol jusqu'à la cuvette de W.-C. avec ou sans chasse d'eau, certains des ménages n'employant aucun dispositif particulier (Tab. 1).

Dans ce contexte d'assainissement autonome, il importe de saisir l'impact sanitaire que peut avoir le rejet incontrôlé des déjections humaines dans l'espace péridomestique et urbain.

\section{Risques diarrhéiques liés à l'évacuation des matières fécales}

Les données traitées montrent une forte implication de ces systèmes d'assainissement dans la survenue des maladies diarrhéiques à Yaoundé (Tab. 2).

Cette association a été confirmée par les recherches microbiologiques réalisées sur les échantillons de selles diarrhéiques prélevées. Ainsi, ont été trouvés : shigelles, salmonelles, Entamœba Coli, anguillules, trichocéphales et ascaris, connus pour être largement impliqués dans l'étiologie des diarrhées infectieuses dans les pays en développement. À Yaoundé, les parasites interviennent dans $30 \%$ des cas de diarrhées chez les enfants et les bactéries, dans $5 \%$ des cas. En association avec les autres germes, notamment les bactéries, ils représentent $84 \%$ des causes de diarrhées. Ces quelques données traduisent les risques pathogènes d'un mauvais assainissement des déjections humaines sur la santé des populations. Directement rejetés dans la nature ou sans dispositif d'épuration adéquat, ces excreta provoquent une double contamination bactériologique et parasitologique du sol et des eaux souterraines. À Yaoundé, comme dans d'autres métropoles africaines (Woldemichael, 2004; Audibert, 1982),

\footnotetext{
${ }^{4}$ D'après le récent rapport d'actualisation du schéma directeur d'aménagement de la ville de Yaoundé, sur les 1500000 habitants que comptait la capitale en 2000, 1300 000, soit près de $87 \%$, utilisaient des techniques d'assainissement autonome.
} 
Tableau 1. Dispositifs utilisés par les habitants de Yaoundé pour l'évacuation des excreta.

\begin{tabular}{lcc}
\hline Types & Nombre de ménages concernés & Pourcentage \\
\hline 1. Dispositifs fonctionnant à l'eau & & 17,7 \\
Latrines avec cuvette et chasse d'eau & 537 & 4,2 \\
Latrines sans cuvette mais avec chasse d'eau (à la turque) & 127 & 3,3 \\
\hline 2. Dispositifs fonctionnant à sec & & 65,6 \\
Latrines avec cuvette sans chasse d'eau & 100 & 8,9 \\
Latrines cimentées, sans cuvette ni chasse d'eau & 1989 & 0,2 \\
Latrines non aménagées (terre battue, trou) & 270 & 100 \\
\hline 3. Sans dispositif & & \\
Nature (brousse, rivière...) & 3031 & \\
\hline Total & & \\
Données manquantes : 3 & & \\
\hline
\end{tabular}

Source : enquête PERSAN / Diarrhées infantiles à Yaoundé - IRD, UR093, mai-juin 2002.

Tableau 2. Pourcentage des diarrhées à Yaoundé selon les dispositifs d'assainissement des matières fécales.

\begin{tabular}{lcc}
\hline Types & Nombre de ménages concernés & Pourcentage de diarrhées \\
\hline 1. Dispositifs fonctionnant à l'eau & & \\
Latrines avec cuvette et chasse d'eau & 43 & 9,8 \\
Latrines sans cuvette mais avec chasse d'eau (à la turque) & 11 & 2,5 \\
\hline 2. Dispositifs fonctionnant à sec & 19 & 4,3 \\
Latrines avec cuvette sans chasse d'eau & 298 & 68,2 \\
Latrines cimentées, sans cuvette ni chasse d'eau & 64 & 14,6 \\
Latrines non aménagées (terre battue, trou) & 2 & 0,4 \\
\hline 3. Sans dispositif & 437 & 100 \\
Nature (brousse, rivière...) & & \\
\hline Total & & \\
\hline
\end{tabular}

les pathologies bactériennes associées à un mauvais assainissement des urines et des matières fécales sont les shigelloses, les amibiases et les salmonelloses, qui sont dues à des parasites à longue durée de vie dans le sol (de 2 mois à 3 ans). Les parasitoses les plus répandues en cas de contamination fécale sont les helminthiases intestinales humaines. Les œufs d'helminthes rejetés dans les selles peuvent survivre plusieurs années à la surface du sol, surtout s'il est humide et chaud, comme c'est le cas des sols et des espaces non revêtus de Yaoundé. Le volet environnemental de cette étude a montré que la contamination se fait soit directement par ingestion de kystes ou d'œufs présents dans l'eau des puits et des sources ou dans les aliments souillés, soit par simple contact cutané avec le parasite qui se trouve dans le sol mouillé; ces résultats sont proches de ceux de Howard et al. (2000) et de Dorrier-Apprill (1993).

S'agissant de la transmission féco-orale, les nématodes sont ici les parasites les plus fréquemment rencontrés (ascaris et trichocéphales). Ils abondent à l'état d'œufs dans le sol, d'autant plus facilement que la plupart des voies et des terrains urbains ne sont pas couverts d'une couche de bitume imperméable. Lors des pluies, la terre humide et tiède qui procède de l'ambiance climatique de Yaoundé constitue un milieu de prédilection pour leur développement. En effet, les femelles pondent des œufs éliminés avec les excreta. Lorsqu'ils sont ingérés directement par l'homme via une eau ou des aliments souillés, ces oeufs donnent naissance à des adultes qui se développent dans l'intestin, où ils occasionnent des gastroentérites.

La transmission transcutanée, quant à elle, concerne les anguillules et les ankylostomes, des vers qu'on trouve dans le sol sous la forme de larves. Ils pénètrent dans le corps humain par la peau. L'homme contracte ces deux parasites en marchant ou en jouant pieds nus dans la boue ou dans les rigoles (Menard, 1996).

\section{Des variations intraurbaines d'incidence de diarrhées}

Les risques de diarrhées réellement encourus par les populations dépendent fondamentalement des dispositifs utilisés pour évacuer leurs urines et leurs matières fécales. 
Tableau 3. Répartition des diarrhées par type de quartier à Yaoundé et selon les dispositifs d'évacuation des matières fécales.

\begin{tabular}{|c|c|c|c|c|c|c|c|c|}
\hline \multirow[b]{2}{*}{ Types de dispositifs } & \multicolumn{3}{|c|}{ Quartier aménagé } & \multicolumn{4}{|c|}{ Quartier spontané } & \multirow{2}{*}{ Total } \\
\hline & $\begin{array}{l}\text { populaire } \\
\text { planifié }\end{array}$ & $\begin{array}{l}\text { populaire } \\
\text { administré }\end{array}$ & $\begin{array}{l}\text { résidentiel } \\
\text { moderne }\end{array}$ & central & péricentral & $\begin{array}{l}\text { de front } \\
\text { urbain }\end{array}$ & $\begin{array}{l}\text { en voie } \\
\text { d'intégra- } \\
\text { tion }\end{array}$ & \\
\hline \multicolumn{9}{|l|}{ Dispositifs fonctionnant à l'eau } \\
\hline \multicolumn{9}{|c|}{ Latrines avec cuvette et chasse d'eau } \\
\hline \multirow{3}{*}{ ménages munis } & 195 & 80 & 33 & 81 & 98 & 35 & 15 & 537 \\
\hline & $17,7 \%$ & $14,9 \%$ & $6,2 \%$ & $15,1 \%$ & $18,3 \%$ & $6,5 \%$ & $2,8 \%$ & $100 \%$ \\
\hline & 10 & 7 & 2 & 8 & 11 & 4 & 1 & 43 \\
\hline cas de diarrhées & $23,2 \%$ & $16,3 \%$ & $4,6 \%$ & $18,6 \%$ & $25,6 \%$ & $9,3 \%$ & $2,4 \%$ & $100 \%$ \\
\hline \multicolumn{9}{|c|}{ Latrines sans cuvette mais avec chasse d'eau (à la turque) } \\
\hline \multirow{3}{*}{ ménages munis } & 66 & 9 & 10 & 16 & 24 & 2 & 0 & 127 \\
\hline & $51,9 \%$ & $7,1 \%$ & $7,9 \%$ & $12,6 \%$ & $18,9 \%$ & $1,6 \%$ & $0 \%$ & $100 \%$ \\
\hline & 7 & 0 & 2 & 1 & 1 & 0 & 0 & 11 \\
\hline cas de diarrhées & $63,6 \%$ & $0 \%$ & $18,2 \%$ & $9,1 \%$ & $9,1 \%$ & $0 \%$ & $0 \%$ & $100 \%$ \\
\hline \multicolumn{9}{|l|}{ Dispositifs fonctionnant à sec } \\
\hline \multicolumn{9}{|c|}{ Latrines avec cuvette sans chasse d'eau } \\
\hline \multirow{3}{*}{ ménages munis } & 3 & 5 & 4 & 40 & 37 & 13 & 8 & 100 \\
\hline & $3,0 \%$ & $5,0 \%$ & $4,0 \%$ & $40,0 \%$ & $37,0 \%$ & $13,05 \%$ & $8,0 \%$ & $100 \%$ \\
\hline & 0 & 0 & 0 & 3 & 12 & 3 & 1 & 19 \\
\hline cas de diarrhées & $0 \%$ & $0 \%$ & $0 \%$ & $15,8 \%$ & $63,2 \%$ & $15,8 \%$ & $5,3 \%$ & $100 \%$ \\
\hline \multicolumn{9}{|c|}{ Latrines cimentées, sans cuvette ni chasse d'eau } \\
\hline \multirow{3}{*}{ ménages munis } & 80 & 184 & 103 & 510 & 675 & 248 & 189 & 1989 \\
\hline & $4,0 \%$ & $9,2 \%$ & $5,2 \%$ & $25,6 \%$ & $33,9 \%$ & $12,5 \%$ & $9,5 \%$ & $100 \%$ \\
\hline & 12 & 27 & 6 & 65 & 128 & 42 & 18 & 298 \\
\hline cas de diarrhées & $4,1 \%$ & $9,1 \%$ & $2,0 \%$ & $21,9 \%$ & $42,9 \%$ & $14,1 \%$ & $6,1 \%$ & $100 \%$ \\
\hline \multicolumn{9}{|c|}{ Latrines non aménagées (terre battue, trou) } \\
\hline \multirow{3}{*}{ ménages munis } & 5 & 26 & 8 & 95 & 70 & 43 & 23 & 270 \\
\hline & $1,8 \%$ & $9,6 \%$ & $2,9 \%$ & $35,2 \%$ & $25,9 \%$ & $15,9 \%$ & $8,5 \%$ & $100 \%$ \\
\hline & 2 & 7 & 2 & 17 & 23 & 7 & 6 & 64 \\
\hline cas de diarrhées & $3,1 \%$ & $10,9 \%$ & $3,1 \%$ & $26,6 \%$ & $35,9 \%$ & $10,9 \%$ & $9,4 \%$ & $100 \%$ \\
\hline \multicolumn{9}{|l|}{ Pas de dispositif } \\
\hline \multirow{4}{*}{$\begin{array}{l}\text { Pot / } \\
\text { broussaille / } \\
\text { rivière }\end{array}$} & 0 & 2 & 0 & 0 & 5 & 1 & 0 & 8 \\
\hline & $0 \%$ & $25,0 \%$ & $0 \%$ & $0 \%$ & $62,5 \%$ & $12,5 \%$ & $0 \%$ & $100 \%$ \\
\hline & 0 & 0 & 0 & 0 & 1 & 1 & 0 & 2 \\
\hline & $0 \%$ & $0 \%$ & $0 \%$ & $0 \%$ & $50 \%$ & $50 \%$ & $0 \%$ & $100 \%$ \\
\hline
\end{tabular}

Seuil de signification : $5 \%$.

Source : enquête PERSAN / Diarrhées infantiles à Yaoundé - IRD, UR093, mai-juin 2002.

À Yaoundé, les niveaux de risques sont variables selon les types de quartiers urbains (Tab. 3).

Les faibles proportions de maladies diarrhéiques se rencontrent dans les quartiers où les ménages utilisent les dispositifs fonctionnant à l'eau (Fig. 2). Il s'agit des quartiers résidentiels modernes et populaires planifiés, où fosses septiques et fosses à chasse manuelle sont couramment utilisées.

En effet, construits sur des terrains viabilisés, les logements ici ont très tôt bénéficié de réseaux intégrés d'évacuation des eaux usées et de vannes. C'est notamment le cas dans les camps SIC ${ }^{5}$ de Mendong, Cité verte et Biyem Assi, où les déjections provenant des immeubles collectifs et des villas des zones pavillonnaires sont collectées dans les fosses septiques et canalisées vers les stations d'épuration (Agendia et al., 2001). Bien qu'en dysfonctionnement, ces stations continuent de recevoir urines et matières fécales, évitant ainsi leur rejet incontrôlé. Cependant, le défaut d'entretien ou de maintenance fait que,

5 Société immobilière du Cameroun. dans certaines cités, ces réseaux frappés par l'usure et par l'âge se détériorent, libérant ainsi leurs contenus infects qui se déversent sur la chaussée et dans les caniveaux (Photo 1). C'est ce qui justifie les cas de diarrhées diagnostiqués et les proportions de 6,1\%, légèrement supérieures à celles de 5,1\% relevées dans les quartiers résidentiels du Lac, de Ngousso, de Santa Barbara ou de Bastos, dans lesquels les résidents n'attendent pas les pouvoirs publics pour prendre eux-mêmes en charge la maintenance de leurs installations.

Toutefois, l'utilisation des dispositifs fonctionnant à l'eau n'est pas l'exclusivité des quartiers aménagés. On les trouve également dans les quartiers spontanés et de front urbain, mais en faible proportion : $4,4 \%$ dans les quartiers en voie d'intégration, $10,8 \%$ dans les quartiers de front urbain et $13,5 \%$ dans les quartiers péricentraux (Tab. 4).

Mis en relation avec la prévalence des diarrhées, ces trois types de tissu urbain présentent des taux disparates: $11,4 \%$ dans les quartiers de front urbain, $46,9 \%$ dans les quartiers spontanés centraux. Ce taux assez élevé par rapport aux quartiers aménagés/planifiés est à rapprocher 

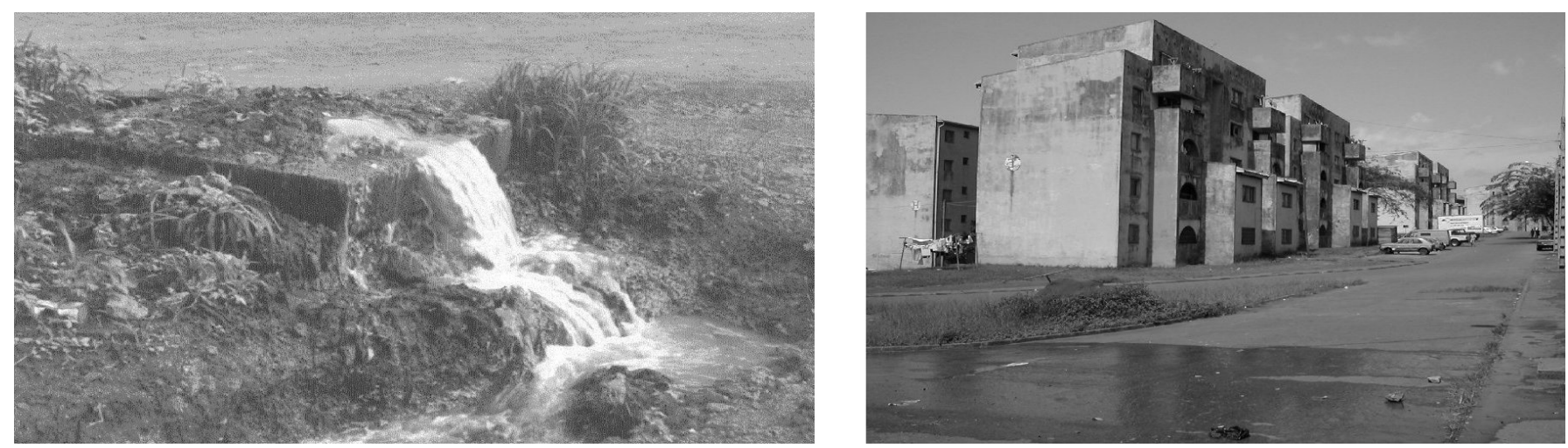

Photo 1. Débordement des eaux usées et de matières fécales à la Cité verte. On pourrait penser à une chute dans une section de cours d'eau ! L'image à gauche, datant de plus d'une décennie, traduit la situation encore visible dans ce quartier. La station d'épuration ayant cessé de fonctionner quelques heures seulement après son inauguration, les eaux usées ont empli les canalisations au point de faire sauter la borne de connexion, libérant ainsi son contenu infect dans la nature et sur la chaussée, comme on s'en aperçoit au premier plan de l'image de droite. (Clichés Tang, 1990, et Nguendo Yongsi, 2004.)

Tableau 4. Dispositifs d'évacuation des matières fécales rencontrés dans les quartiers de Yaoundé.

\begin{tabular}{lcc}
\hline Types de quartiers & Dispositifs fonctionnant à l'eau & Dispositifs fonctionnant à sec \\
\hline Quartiers planifiés et résidentiels & $74,40 \%$ & $25,60 \%$ \\
Zones de recasement & $27,20 \%$ & $72,80 \%$ \\
Quartiers spontanés centraux & $14,90 \%$ & $85,10 \%$ \\
Quartiers spontanés péricentraux & $13,50 \%$ & $86,50 \%$ \\
Quartiers de front urbain & $10,80 \%$ & $89,20 \%$ \\
Quartiers en voie d'intégration & $4,40 \%$ & $95,60 \%$ \\
\hline
\end{tabular}

Source : enquête PERSAN / Diarrhées infantiles à Yaoundé - IRD, UR093, mai-juin 2002.

de la qualité technique des dispositifs présents. Contrairement aux quartiers planifiés, où les fosses septiques répondent aux normes de construction et de fonctionnement, dans les quartiers spontanés d'Etoa Meki, Melen, Briqueterie, etc., ces fosses sont réalisées par des ouvriers journaliers ignorant les exigences de l'art. Ici, en effet, les fosses septiques n'ont pas d'élément épurateur du genre lit bactérien. Les matières fécales sont envoyées dans le puisard sans prétraitement. La fosse septique fonctionnant alors uniquement comme un digesteur, il s'en dégage des odeurs qui attirent les insectes. Sous l'effet de la pression, les parois de la fosse se fissurent et laissent échapper les effluents dans la nature.

Les proportions les plus élevées de diarrhées ont été recensées dans les quartiers où les ménages recourent davantage aux dispositifs fonctionnant à sec (Fig. 3). Une fois encore, les quartiers spontanés et leurs semblables (les zones de recasement) sont en tête. N'estce pas là qu'on utilise le plus couramment les latrines non améliorées, dont les descriptions montrent que les risques sanitaires sont réels et les risques de contamination grands : propagation d'odeurs nauséabondes, afflux des mouches sur les déjections répandues sur le sol ou dans les cours et les ruelles non revêtues, pollution de la nappe phréatique $^{6}$ !

Ainsi, d'un quartier spontané à l'autre, les taux de diarrhées varient. Assez modérés dans les zones de recasement et dans les quartiers en voie d'intégration, ils sont nettement plus forts dans les quartiers spontanés centraux et péricentraux. Dans ces deux derniers types de quartiers, en effet, l'évaluation de la gestion des matières fécales à partir de fosses simples représente un aspect majeur du problème général de l'insalubrité. Nous avons vu qu'au niveau de la construction, toutes ces fosses avaient des parois sans protection. Plus grave, les propriétaires font preuve d'une incroyable légèreté dans la manipulation des

6 Trois dispositifs de latrines à sec existent : (i) à fosse sèche ou à fond perdu - il s'agit de simples trous sans maçonnerie, de 2 à $5 \mathrm{~m}$ de profondeur, dans lesquels on verse des substances chimiques pour baisser le volume ; (ii) à fosse forée - recouvertes d'une dalle de béton, leur profondeur varie de 2 à $10 \mathrm{~m}$ et elles se composent de deux compartiments (douche et toilettes). Leur vidange n'est pas effectuée en raison des difficultés d'accès et des coûts élevés que nécessite l'opération ; (iii) les fosses d'infiltration à structure maçonnée - situées à l'intérieur des habitations, leur fond est perméable (en terre) et permet l'infiltration directe des eaux résiduaires (Megno, 2000). 


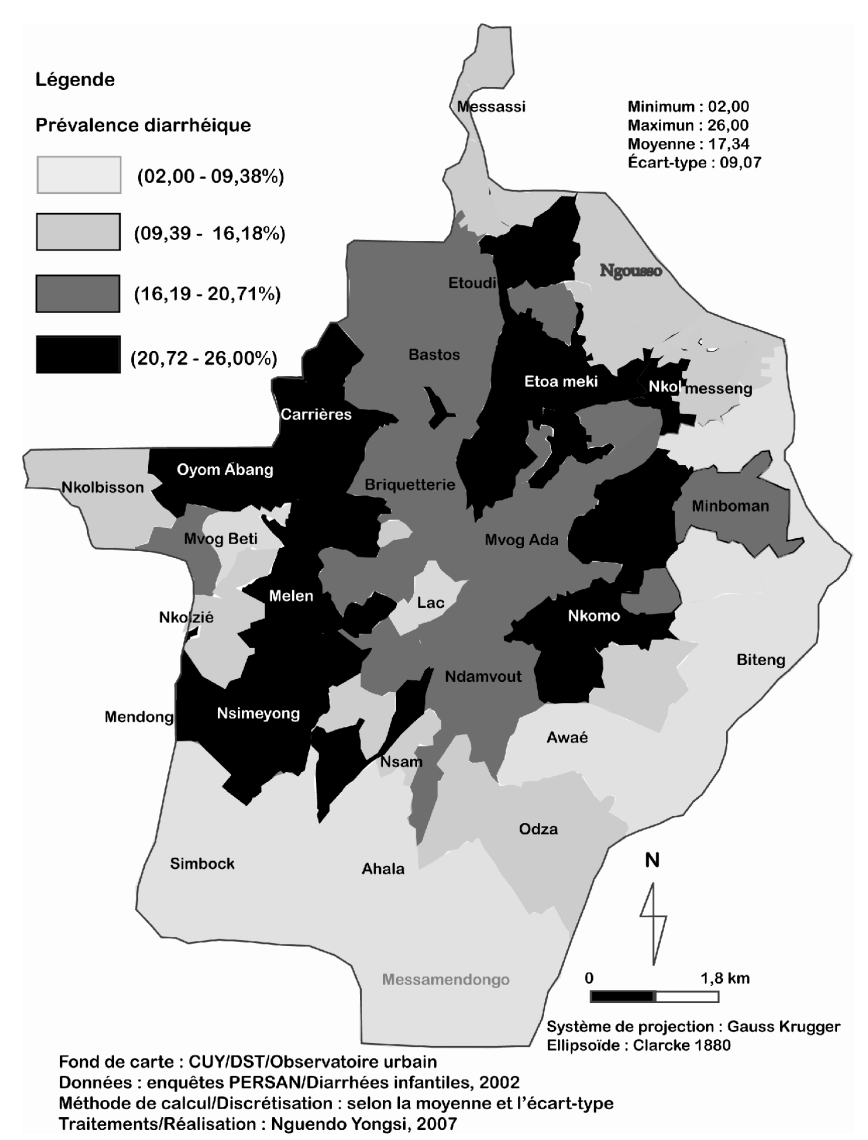

Fig. 2. Pourcentage des diarrhées associées à l'utilisation des dispositifs fonctionnant à l'eau (fosses septiques avec chasse d'eau) à Yaoundé.

excreta : les fosses sont vidées de leur contenu derrière les habitations (dans les rigoles ou dans les cages à porcs). La vidange étant manuelle, ceux qui l'exécutent sont parfois tachés par les matières fécales. Des odeurs nauséabondes infectent l'air, et des filaments de matières fécales forment des réseaux anastomosés entre les habitations - filaments qui disparaissent progressivement grâce aux semelles des passants ${ }^{7}$, aux sabots des moutons et des chèvres, et aux ongles des poules et des canards tenus en laisse. Ces excréments peuvent ainsi être diffusés partout, même à l'intérieur des habitations, et contaminer directement les ustensiles. Indirectement, la contamination peut être réalisée par les insectes habituels, qui complètent ainsi la chaîne de transmission pathologique.

La situation des quartiers spontanés de bas-fonds habités, comme Etam Bafia, Fanta Citron, Elig Edzoa, Mfandena, Mokolo Elobi, Ndjong Melen, est particulière. La majorité des parcelles sont ici équipées de latrines rustiques (Akongwié, 1999). La surface de la nappe aquifère étant proche de la surface du sol, le risque de contamination fécale de l'eau souterraine se trouve accrû lors des

\footnotetext{
${ }^{7}$ Les pieds nus des enfants imprudents étant loin d'être les moins agissants.
}

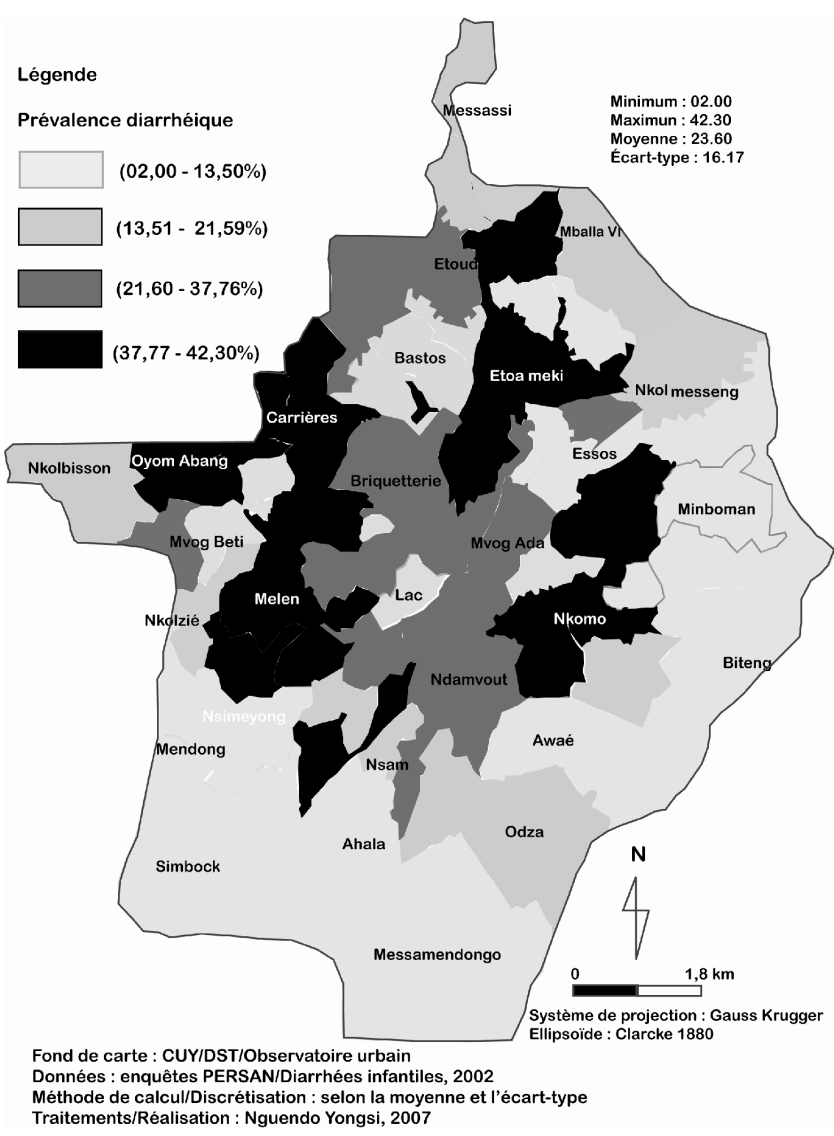

Fig. 3. Pourcentage des diarrhées associées à l'utilisation des dispositifs fonctionnant à sec (latrines traditionnelles) à Yaoundé.

fréquentes inondations. À l'occasion, celles-ci provoquent le débordement des fosses sur les parcelles. Lors du retrait des eaux, ou à l'averse suivante, de nombreux microorganismes d'origine fécale peuvent ainsi se retrouver disséminés sur les sols nus, ou entraînés dans le sous-sol et contaminer la nappe. De tels espaces, on s'en doute bien, constituent des zones à risques de maladies infectieuses dont les diarrhées ne sont pas les moindres. Les 19,7 \% de diarrhées relevés dans les quartiers spontanés péricentraux de la ville se localisent dans ces bas-fonds aux dispositifs d'évacuation sommaires (Photo 2).

\section{Conclusion}

L'analyse des modes de gestion des eaux usées comme facteur de risque associé aux diarrhées et celle des variations intraurbaines d'incidence de ces diarrhées permet de montrer l'intérêt de réaliser une géographie des états de santé à l'échelle réduite des quartiers en milieu urbain tropical. Du point de vue du facteur de risque que nous avons considéré (modes d'évacuation des urines et des matières fécales), cette étude est un exemple qui présente un aspect majeur du phénomène de croissance 

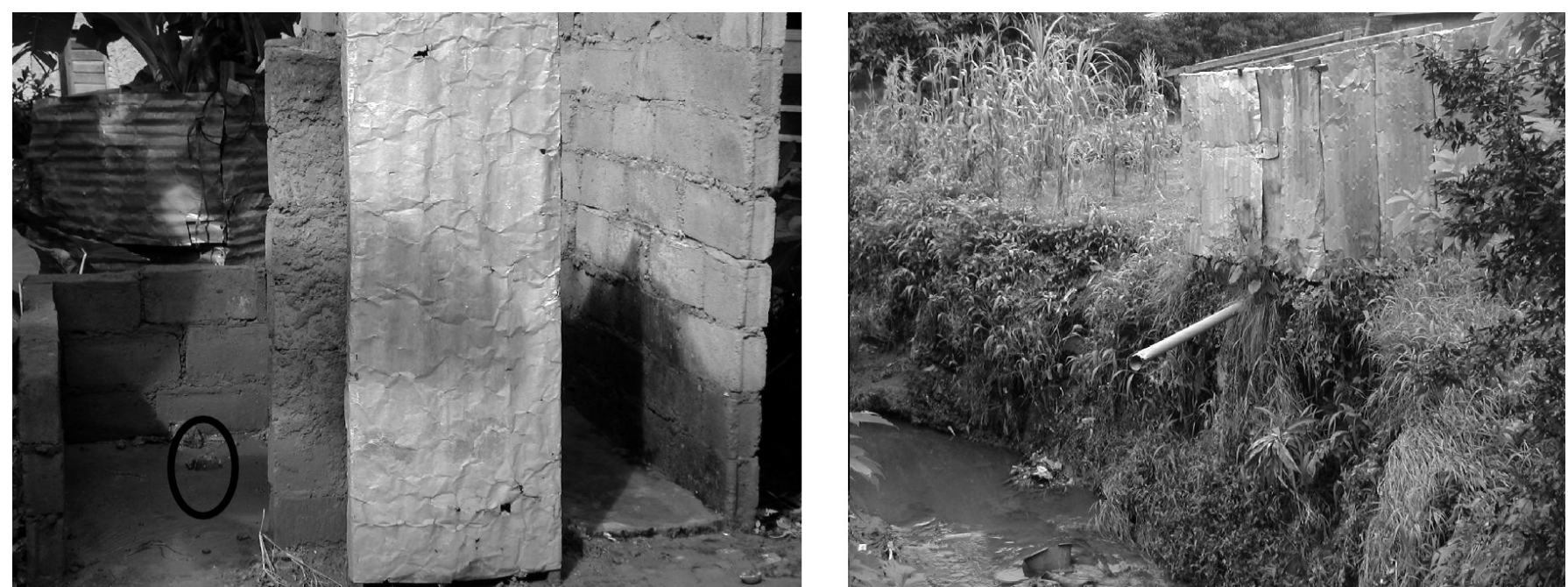

Photo 2. Dispositifs d'évacuation des matières fécales dans les quartiers d'habitat spontané de Yaoundé. À gauche, un simple trou réalisé dans le sol sans protection aucune; à droite, urines et matières fécales sont rejetées dans la rivière à l'aide d'un tuyau de PVC de $10 \mathrm{~cm}$ de diamètre. La fosse en elle-même est une petite excavation étanche, dallée, de 10 à $15 \mathrm{~cm}$ de diamètre. (Clichés Nguendo Yongsi, 2004.)

des villes en développement, celui de la relation avec la pathologie et son impact sur le niveau de santé des citadins. Le choix du niveau contextuel pour l'analyse permet de voir que les quartiers urbains ne sont pas tous intensément soumis aux mêmes niveaux d'atteinte diarrhéique. Les figures 2 et 3 montrent que ce facteur de risque joue variablement $d^{\prime}$ un sous-espace urbain à un autre, traduisant par là l'enseignement majeur qu'en milieu urbain africain - et ceci, contrairement à la littérature existante -, toutes les misères se trouvent rarement réunies en un seul endroit. La possession de telles données qui présentent les populations les plus à risques de maladies diarrhéiques dans la ville peut être utile pour les aménageurs et autres professionnels de la santé pour l'adoption des stratégies appropriées et efficaces. En clair, cela permet de penser que des solutions sont possibles si l'on veut venir à bout de cette forme d'urbanisation à l'impact négatif sur la santé des citadins.

Ce travail est loin d'être complet. Bien que la méthode d'analyse choisie (association ou analyse bivariée), qui, elle, relève de notre approche d'interprétation (niveau contextuel), obéisse à la démarche écoépidémiologique recommandable pour ce type de travail, il apparaît difficile de lier l'existence des maladies diarrhéiques à Yaoundé aux seuls modes de gestion des matières fécales. D'autres facteurs de risques existent qui, eux aussi, contribuent au développement des diarrhées. Aussi, seule une approche multivariée incluant de nombreux facteurs peut permettre de déterminer le poids de chacun d'eux dans la prévalence des diarrhées dans la ville.

\section{Remerciements}

Ce travail fait partie d'une étude interdisciplinaire (géographie, épidémiologie, médecine) de l'IRD, centrée sur la santé en milieu urbain. Nous voulons donc remercier l'Institut de recherche pour le développement pour l'appui matériel et logistique apporté pour sa réalisation. Notre gratitude va également envers la direction des Services techniques de la communauté urbaine de Yaoundé pour avoir mis à notre disposition le fichier numérisé de la ville. Nous remercions vivement les évaluateurs et lecteurs de ce manuscrit, dont les avis et les suggestions nous ont permis d'éviter certaines maladresses.

\section{Références}

Addy, P.A., Antepim, G., Frimpong, E.H., 2004. Prevalence of pathogenic Escherichia coli and parasites in infants with diarrhoea in Kumasi, Ghana, East Afr. Med. J., 81, 7, 353-357.

Agendia L.P., et al.. 2001. Collecte, épuration et évacuation des eaux usées dans les lotissements SIC-MAETUR de Yaoundé, in Eno Belinga, S.M., Vicat, J.-P. (Eds), Yaoundé : une grande métropole africaine au seuil du troisième millénaire, Yaoundé, PUY, 169-178.

Akongwié, P., 1999. Marshy Settlements in Yaoundé. Mémoire de DIPLEG, ENS, Yaoundé.

Amat-Roze, J.M., 1983. L'urbanisation dans les pays tropicaux, Médecine tropicale, 43, 4, 379-384.

Audibert, M., 1982. Statistiques sanitaires et indicateurs de santé dans les pays en voie de développement, Revue d'épidémiologie et de santé publique, 30, 437-450.

Bley, D., Champaud, J., et al.. (Eds), 1997. Urbanisation et environnement dans les PVD, Paris, SEH/ORSTOM. 
Colson, J.-C., 1808. Essai sur la diarrhée. Thèse de médecine, Faculté de médecine, Paris.

Dennehy, P.H., Dennehy, P.H., 2005. Acute diarrhoeal disease in children: epidemiology, prevention, and treatment, Infect. Dis. Clin. North. Am., 19, 3, 585-602.

Dorier-Aprill, E., 1993. Environnement et santé à Brazzaville (Congo), de l'écologie urbaine à la géographie sociale. Thèse de doctorat NR, Université de Paris X - Nanterre.

Dufour, A., 1814. Dissertation sur la diarrhée idiopathique. Thèse de médecine, Faculté de médecine, Paris.

Dupont, C., 1952. Les Diarrhées de l'enfant, Paris, IPSEN.

Ferrière, S., 1992. Diarrhées aiguës de l'enfant : étude épidémiologique, clinique et microbiologique. Thèse d'exercice en médecine, Université de Tours.

Galien, 1854. CEuvres anatomiques, physiologiques et médicales, trad. et éd. par le Dr Charles Daremberg, Paris, J.-B. Baillière, tome 1 .

Gentilini, M., Duflo, B., 1986. Médecine tropicale, Paris, Flammarion.

Giacottino, J.C., 1979. La ville tropicale et ses problèmes d'environnement, Cahiers d'outre-mer, 125, 22-37.

Howard, P., et al., 2000. Bacterial, viral and parasitic aetiology of paediatric diarrhoea in the Highlands of Papua-New Guinea, J. Trop. Pediatr., 46, 10-14.

Imbert, P., 2001. Prise en charge des diarrhées aiguës de l'enfant en milieu tropical, Médecine tropicale, 61, 3, 226-230.

Kuété, M., 1977. Étude géomorphologique du massif de Yaoundé. Thèse de doctorat de $3^{\mathrm{e}}$ cycle en géographie, Université de Bordeaux 3.

Megno Ouambo, L., 2000. Évacuation des eaux usées et excréta humains dans les quartiers à habitat spontané : cas des quartiers Mokolo et Madagascar à Yaoundé. DESS en sciences de l'environnement, Faculté des sciences, Université de Yaoundé I, Cameroun.

Reçu le 5 novembre 2006. Accepté le 8 novembre 2007.
Menard, B., 1996. Contribution géographique à l'étude de la santé dans le tiers-monde. Exemple de la trilogie pathologique "diarrhéemalnutrition-affections respiratoires " observée chez les jeunes enfants. Thèse de doctorat NR, Université de Caen.

Ministère du Plan et de l'Aménagement du territoire [MINPAT], 1998. Enquête démographique et de santé (EDSC), Yaoundé, Cameroun.

Ngwe, E., 1999. La Gestion des déchets par les ménages : un problème crucial d'environnement urbain. L'exemple de Yaoundé (Cameroun). Cours de la chaire Quetelet, Université catholique de Louvain, Institut de démographie, Louvain-laNeuve, Belgique.

Nielly, M., 1881. Éléments de pathologie exotique, Paris, A. Delahaye et E. Lecronier.

OMS, 2001. Assainissement et diarrhée, Agir contre les infections, $2,7,1-2$.

OMS, 2006. http://www.who.org/ (page consultée le 4 octobre 2006).

Ondoua, O., 1992. La Transition démographique au Cameroun et le cas particulier de Yaoundé. Thèse de doctorat en géographie, Université de Bordeaux 3.

Snyder, J.D., Merson, M.H., 1982.The magnitude of the global problem of acute diarrhoeal disease: a review of active surveillance data, Bull. WHO, 60, 605-613.

Tanawa, E., Djeuda Tchapnga, H.B., Ngnikam, E., Temgoua, E., 2002. Eau, environnement et santé : d'énormes difficultés à surmonter, in Giroult, E., Seux, R., Eau, environnement, santé : un enjeu majeur pour le XXI siècle, Rennes, ENSP, 362-371.

Woldemicael, G., 2004. Diarrhoeal morbidity among young children in Eritrea: environmental and socio-economic determinants, J. Health Popul. Nutr., 19, 2, 83-90. 